of the disorder responsible for causing RPL and its overwhelmed complement activation recognized as a major pathogenic mechanism. Autoantibodies against complement component 1q subcomponent (aC1q) have been shown to associate with complement activation in primary APS, but the relevance of aC1q in RPL is still unclear. We hypothesized that aC1q would be associated with the pathogenesis of RPL in patients with or without APS, especially in RPL of unknown etiology.

Objectives: The aim of this study was to explore the significance of aC1q in RPL. Methods: As a clinical study, we conducted a retrospective cross-sectional study comprising a total of 134 patients with RPL of unknown etiology, 27 with obstetric APS (OAPS), 14 parous patients with connective tissue disease (CTD) without historical obstetric/thrombotic complications and 17 parous healthy controls (HC). Serum levels of aC1q were measured using a solid-phase ELISA (Buhlmann Laboratories AG, Switzerland) and defined as positive using cut-off value of more than $15 \mathrm{U} / \mathrm{mL}$ according to the manufacturer. In murine model, 8-12 week-old female BALB/c mice were mated with isolated males and the presence of vaginal plug was defined as day 1 of pregnancy. Mice were treated with intravenous injections of anti-mouse C1q monoclonal antibody (JL-1), isotype control IgG2b or PBS. To block $\mathrm{C} 5$ a receptor $(\mathrm{C} 5 \mathrm{aR})$, mice were intravenously pre-treated with anti-C5aR antibody, 30 minutes before the injection of $\mathrm{JL}-1$ on day 8 . Mice were sacrificed on day 16 of pregnancy and fetal resorption ratios, weight of fetuses and placentas, serum levels of $\mathrm{C} 3 \mathrm{a}$ and immunohistochemical staining of complement components on placental tissue were compared among each group.

Results: Among RPL, OAPS, CTD and HC, $47(35 \%), 8(30 \%), 3(21 \%)$ and $2(12 \%)$ were positive for aC1q, respectively. In RPL patients, aC1q was more prevalent $(p<0.05)$ and its titer was significantly higher than in $\mathrm{HC}$ (median and interquartile range [IQR] 12 [8-21] vs. 0 [0-4.3], $p<0.0001$ ) (Figure 1). In murine model, fetal resorption ratio was higher $(p<0.01)$, weight of fetuses and placentas lower $(p<0.05)$, and serum levels of C3a higher $(p<0.01)$ in mice treated with $\mathrm{JL}-1$ than in control mice. Immunohistological findings showed that complement components were more deposited on placenta in $\mathrm{JL}-1$ treated mice than in control mice. Furthermore, the additional blockade of $\mathrm{C} 5 \mathrm{aR}$ cancelled the pathogenic changes in $\mathrm{JL}-1$ treated mice.

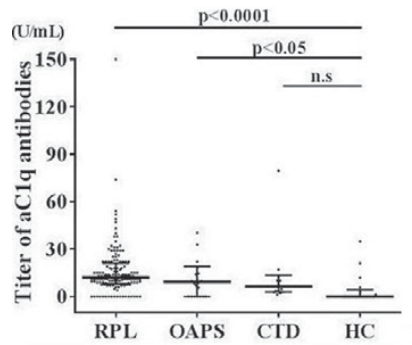

Figure 1. Titers of anti-Clq antibodies $(\mathrm{aC} 1 \mathrm{q})$ among recurrent pregnancy loss (RPL), obstetric antiphospholipid syndrome (OAPS), connective tissue disease (CTD) and healthy controls (HC) groups.

The titers of aClo were significantly higher in RPL and OAPS compared to HC group (Dunn test). Honizontal bars show median and whiskersindicate the first and third quartiles.

Conclusions: Clinical findings showed that aC1q could be relevant to RPL. Moreover, we have established aC1q induced pregnancy loss model mice. Our study indicates that $\mathrm{aC} 1 \mathrm{q}$ has a pathophysiologic role in RPL and that anticomplement therapy might be effective for at least some groups of patients with RPL for whom specific treatment remains to be established.

Disclosure of Interest: None declared

DOI: 10.1136/annrheumdis-2017-eular.1008

\section{AB1140 WHO DISABILITY ASSESSMENT SCHEDULE 2.0 IS RELATED TO UPPER AND LOWER EXTREMITY SPECIFIC QUALITY OF LIFE}

K.S. Park, Y.-H. Cheon. Gyeongsang National University, Jinju, Korea, Republic Of

Background: Musculoskeletal complaints influence disability, but the relative contribution of concurrent upper and lower extremity health-related quality of life (HRQOL) on patient perceptions of disability is unclear.

Objectives: We evaluated whether two disease specific quality of life instruments (DASH and WOMAC) reflect a patient's perception of general disability using the WHO Disability Assessment Schedule 2.0 (WHODAS 2.0) and determined whether disability components are explained by upper and lower extremity HRQOL.

Methods: We recruited 421 randomly chosen participants 50 years or older without stroke, cancer, or history of surgery for musculoskeletal disease who participated in the Namgang Cohort. Upper extremity HRQOL was determined with the DASH score and lower extremity HRQOL with the WOMAC; as a measure of disability, we obtained WHODAS 2.0 component. Multiple regression modeling was used to assess the relative contributions made by upper and lower extremity HRQOL to disability.

Results: Most patients reported knee pain (61.0\%), shoulder (17.1\%), elbow $(28.5 \%)$ and hand $(56.1 \%)$. Mean WHODAS 2.0 total score was $28.06(S D=14.2)$, corresponding to mild to moderate disability and WOMAC and DASH scores were $23.2(S D=22.1)$ and $22.4(S D=19.3)$. When adjusted for age, sex, level of education, spouse, self rated health, hypertention, DM and depression, the DASH total score was correlated with the getting around $(\beta=0.137, p=0.032)$ and social participation $(\beta=0.226, p<0.001)$ and the WOMAC total score was correlated with the getting around $(\beta=0.362, p<0.001)$ and social participation $(\beta=0.289$, $\mathrm{p}<0.001$ )

Conclusions: We found that in a community-based population, perceived actibity limitation and social participation were associated with upper and lower extremity HQRQOL. Since the WHODAS 2.0 does not target a specific disease (as oppose to the DASH, WOMAC), it can be used to compare disabilities caused by different diseases.

\section{References:}

[1] PösI M, Cieza A, Stucki G. Psychometric properties of the WHODASII in rehabilitation patients. Qual Life Res. 2007;16(9):1521-31.

[2] Silva C, Coleta I, Silva AG, Amaro A, Alvarelhão J, Queirós A, Rocha N. Adaptation and validation of WHODAS 2.0 in patients with musculoskeletal pain. Rev Saude Publica. 2013 Aug;47(4):752-8.

[3] Tazaki M, Yamaguchi T, Yatsunami M, Nakane Y. Measuring functional health among the elderly: development of the Japanese version of the World Health Organization Disability Assessment Schedule II. Int J Rehabil Res. 2014 Mar;37(1):48-53.

Acknowledgements: This work was supported from the Ministry of Agriculture Food and Rural Affairs in Korea.

Disclosure of Interest: None declared

DOI: 10.1136/annrheumdis-2017-eular.4589

\section{AB1141 PREVALENCE OF LONG-TERM STEROID THERAPY: FRENCH DATA}

L. Couvaras, S. Trijau, G. De Lamotte, V. Pradel, T. Pham, P. Lafforgue. APHM, Marseille, France

Background: Corticosteroids are widely used for various diseases, from chronic respiratory conditions to auto-immune disorders. However, there are few epidemiological data about long-term steroid therapy in southern Europe (1, 2, 3).

Objectives: To describe chronic glucocorticoid prescriptions in a large cohort. Methods: Information was collected from a national public health-insurance database that covers 4.1 million individuals and $83 \%$ of the population, in our geographic area of Provence-Alpes-Côte-d'Azur and Corsica, from September 1, 2009 through August 31, 2011. We identified subjects aged of 15 years and over starting glucocorticoid therapy. Chronic glucocorticoid therapy was defined as $\geq 7.5 \mathrm{mg}$ of prednisone equivalent per day during at least 90 days consecutive. We identified the incident cases of long-term glucocorticoid therapy, defined as those prevalent cases who did not fill glucocorticoid prescriptions during the first 6 months of the 24-month study period.

Results: We identified 32,812 patients who were prescribed glucocorticoid therapy, yielding $0.97 \%$ prevalence. Of these 32,812 patients, $14,205(43.3 \%)$ met our definition of incident cases, yielding an incidence of $0.42 \%$ for 18 months in the overall population aged at least 15 years, corresponding to an incidence of long-term glucocorticoid therapy of 2.8/1000 inhabitants/year. Among these incident cases, the most currently prescribed glucocorticoids were prednisolone $(64 \%)$ and prednisone (32\%). Sixty-three per cent of patients received only one type of glucocorticoid while $33 \%$ received two and $5 \%$ received 3 or more of them. The average treatment duration was 270.9 days ( $\mathrm{Cl} 95 \% 267.7-274)$. Most prescriptions $(55,4 \%)$ were initiated by general practitioners. The median prednisone-equivalent dose was $11 \mathrm{mg} /$ day (IQR, 8.8-17.8) and varied very little with age and sex.

Rheumatoid arthritis was the most common disease associated with chronic glucocorticoid prescriptions in this cohort $(30 \%)$, followed by chronic respiratory failure $(21 \%)$, internal medicine diseases such as connectivite tissue diseases, polymyalgia rheumatica or Giant-cell arteritis (21\%), asthma (15\%) and infammatory bowel diseases like ulcerative colitis or Crohn's disease (13\%).

Conclusions: Long-term corticosteroid therapy is frequent in France, its description is close to what is already known in Europe.

\section{References:}

[1] Overman RA, Heh JY, Deal CL. Prevalence of oral glucocorticoid usage in the United States: a general population perspective. Arthritis Care Res (Hoboken) 2013:65:294-8.

[2] Gudbjornsson B, Juliusson UI, Gudjonsson FV. Prevalence of long term steroid treatment and the frequency of decision making to prevent steroid induced osteoporosis in daily clinical practice. Ann Rheum Dis 2002;61:32-6.

[3] Fardet L, Petersen I, Nazareth I. Prevalence of long-term oral glucocorticoid prescriptions in the UK over the past 20 years. Rheumatology 2011;50:198290.

Disclosure of Interest: None declared

DOI: 10.1136/annrheumdis-2017-eular.6792 